\title{
Isotope Effect of Hydrogen in the Bromination of 1,3,5-Trimethoxybenzene with N-Bromosuccinimide in Dimethylformamide
}

\author{
ERIK HELGSTRA N D *
}

Nobel Institute of Chemistry, Stockholm 50, Sweden

\begin{abstract}
The reaction studied apparently involves a rate-determining step in which free bromine is formed from $\mathrm{N}$-bromosuccinimide and hydrobromic acid and a fast product-determining step in which free bromine reacts with the substrate, forming l-bromo-2,4,6-trimethoxybenzene and hydrobromic acid. The free bromine that starts the reaction is generated by a decomposition reaction of $\mathrm{N}$-bromosuccinimide, probably influenced by the solvent dimethylformamide with its slight content of water.

The product-determining step has been investigated in competitive experiments with tritium, and the isotope effect, $k_{\mathrm{T}} / k_{\mathrm{H}}$ was found to be $1.00 \pm 0.03$ at $20^{\circ} \mathrm{C}$. This result is discussed in relation to previous work.
\end{abstract}

T

There are several cases ${ }^{1-5}$ in which primary isotope effects have been observed in electrophilic substitution reactions with sterically hindered aromatic compounds. Although there is some steric hindrance present in the 1,3,5-trimethoxybenzene molecule, it has been shown ${ }^{6}$ that there is no primary isotope effect in the azo coupling reaction between this molecule and the $p$ chlorobenzenediazonium ion. It was of interest to investigate whether or not the substitution reaction of 1,3,5-trimethoxybenzene with bromine would show a primary isotope effect. Myhre ${ }^{3}$ showed that there was a full primary isotope effect in the bromination of tri-t-butylbenzene, although nitration of the same substrate gave no primary isotope effect.

1,3,5-Trimethoxybenzene is so reactive towards the more studied brominating reagents, such as free bromine or acidified hypobromous acid, that it is not possible to use these reagents in an isotope effect study even if a competitive method is used. In searching for a milder reagent, $\mathrm{N}$-bromosuccinimide in a polar solvent was found to be promising.

* Present address: Department of Organic Chemistry, University of Göteborg, Gibraltargatan 5 A, Göteborg S, Sweden. 
There are several indications ${ }^{7-10}$ that the substitution reaction between $\mathrm{N}$-bromosuccinimide and an aromatic nucleus proceeds by a polar mechanism. Preparative experiments showed that the reaction between 1,3,5-trimethoxybenzene and $\mathrm{N}$-bromosuccinimide in dimethylformamide was quantitative with 1-bromo-2,4,6-trimethoxybenzene as the only product. In this medium the rate of the reaction was not too high and it was quite possible to make a competitive investigation of the isotope effect.

\section{EXPERIMENTAL}

Materials used. 1,3,5-Trimethoxybenzene was prepared as described previously. ${ }^{6}$ The crude product was recrystallized four times from petroleum ether. In order to obtain 1,3,5-trimethoxybenzene-2-t with a suitable activity, a small amount of 1,3,5-trimethoxybenzene-2-t with a relatively high activity ${ }^{6}$ was added in the last recrystallization step and the material thus obtained was sublimed in vacuo.

Commercial (Hopkins \& Williams) N-bromosuccinimide was recrystallized from water, dried in vacuo and stored over anhydrous potassium hydroxide at about $5^{\circ} \mathrm{C}$.

Baker's analyzed dimethylformamide was used without further purification. The specified water content was $0.02 \%$.

Other chemicals used in the kinetic observations were all commercial products and used without further purification.

Analysis. The analysis of the starting material and the reaction product was performed on a gas chromatograph, Perkin-Elmer Model $116 \mathrm{E}$. Conditions: $60 \mathrm{~cm}$ column "O" (silicon grease on chromosorb), int. diam. $4 \mathrm{~mm}$, temp. $192-194^{\circ} \mathrm{C}$, carrier gas $\mathrm{He}$, flow rate $120 \mathrm{ml} / \mathrm{min}$.

The extent of the reaction in the competitive experiments was determined by analyzing the reaction solution, and the ratio of the heights of the two peaks belonging to remaining 1,3,5-trimethoxybenzene and 1-bromo-2,4,6-trimethoxybenzene was measured. This ratio was calibrated by the same type of ratio obtained from the analysis of a reference solution with known amounts of 1,3,5-trimethoxybenzene and 1-bromo-2,4,6-trimethoxybenzene. The mole ratio of the remaining starting material and the product was thus obtained with a relative error less than $\pm 10 \%$ and the calculated extent of the reaction around $90 \%$ can then be given with an absolute error of $\pm 1 \%$.

By analyzing the reaction solution by gas chromatography it was also verified that 1-bromo-2,4,6-trimethoxybenzene was the only product formed.

The isolated starting material and product were to a slight extent contaminated with product and starting material, respectively, and the amounts of these impurities were also determined by gas chromatography. By calibrating with reference solutions as described above, the amounts of impurities in the isolated substances were determined within \pm 0.1 mole $\%$ impurity in the interval $0.2-1$ mole $\%$ impurity. In this way very small corrections of the activity of the isolated substances could be made and the true activity of the pure substances was thus obtained.

Preparative experiment. To a well-stirred solution of 1,3,5-trimethoxybenzene $(5.02$ $\mathrm{g}, 2.98 \mathrm{mmole})$ in $40 \mathrm{ml}$ of dimethylformamide, a solution of $\mathrm{N}$-bromosuccinimide $(5.32$ $\mathrm{g}, 2.99 \mathrm{mmole}$ ) in $60 \mathrm{ml}$ of dimethylformamide was rapidly added. The solution was al. lowed to stand for $20 \mathrm{~min}$ and was then poured into $2.5 \mathrm{l}$ of water. The mixture was cooled to about $5^{\circ} \mathrm{C}$ and allowed to stand over night. The crystals formed were filtered off, washed with water and dried over silica gel. The dry product weighed $7.15 \mathrm{~g}, 97 \%$ of the theoretical yield. The melting point was $99^{\circ} \mathrm{C}$ (determined on a Kofler Hot Stage apparatus) undepressed by 1-bromo-2,4,6-trimethoxybenzene prepared by a reported method.11 According to gas chromatography the only product formed was 1-bromo2,4,6-trimethoxybenzene.

Kinetic observations. It was observed that solutions of $\mathrm{N}$-bromosuccinimide in dimethylformamide became pale yellow; the colour intensity increased with time. By comparing the electronic spectra $(300-600 \mathrm{~m} \mu)$ of this soluticn and of a solution of bromine in dimethylformamide it was made very probable that the colour developed in the $N$. bromosuccinimide solution was due to free bromine. The rate of this decomposition reac-

Acta Chem. Scand. 18 (1964) No. 7 
tion of $\mathrm{N}$-bromosuccinimide in the solvent dimethylformamide increased with temperature, and ultraviolet light had a pronounced effect. Bromine, and perhaps N-bromosuccinimide as well, reacted with the solvent to a small extent. It was found that bromine formed in an $\mathrm{N}$-bromosuccinimide solution immediately disappeared when a reactive substrate such as 1,3,5-trimethoxybenzene was added.

The reaction was followed by taking aliquots from the reaction solution at suitable time intervals, quenching in acidified potassium iodide solution and titrating with thiosulfate solution. Observations on the reaction rate were made with the following substrates: 1,3,5-trimethoxybenzene, 1-bromo-2,4,6-trimethoxybenzene, phenol and $\mathrm{N}$ dimethylaniline. The influence of small amounts of added chloranil, bromine, or silver sulfate was also studied.

Since the reaction is highly exothermic it was very difficult to maintain a constant temperature, especially when runs with higher concentrations of the reactants were made.

A simple kinetic form was not obtained. The total reaction order was somewhat less than one relative to the total content of "active" bromine measured and the rate was approximately independent of the concentration of the substrate. The order was closer to one at lower temperatures. It was also found that the reaction rate depended to a rather great extent on the way in which $\mathrm{N}$-bromosuccinimide was added to the substrates. The rate was about twice as fast when a ten-minutes-old $\mathrm{N}$-bromosuccinimide solution was added to a 1,3,5-trimethoxybenzene solution than when a freshly prepared $N$. bromosuccinimide solution was added to the same solution.

Competitive experiments on preparative scale. Four competitive experiments were carried out.

The reaction was performed at $20.0 \pm 0.2^{\circ} \mathrm{C}$ in a closed system. To a thermostated, well-stirred solution of 1,3,5-trimethoxybenzene-2-t $(2.9-3.1 \mathrm{~g}, 17.2-18.4 \mathrm{mmole})$ in $20 \mathrm{ml}$ of dimethylformamide, a thermostated solution of $\mathrm{N}$-bromosuccinimide $(2.7-3.0$ $\mathrm{g}, 15.2-16.9 \mathrm{mmole}$ ) in $40 \mathrm{ml}$ of dimethylformamide was added during one hour. About $10 \mathrm{~min}$ later, the extent of the reaction was determined by gas chromatography as described above. It was found that the reaction was quantitative within the error limits of the analysis.

The reacted solution was diluted with $50 \mathrm{ml}$ of $2.5 \%$ sodium carbonate solution and continuously extracted for $3 \mathrm{~h}$ with $70 \mathrm{ml}$ of hexane. The extract was cooled to about $5^{\circ} \mathrm{C}$ and allowed to stand over night, whereupon most of the product crystallized. The crystals were filtered off, washed with hexane and air-dried on the filter. The isolated product contained less than 0.8 mole $\%$ of starting material and was used without further purification for the tritium determinations. The filtrate was dried over anhydrous sodium sulfate, concentrated to about $25 \mathrm{ml}$ and the product again allowed to crystallize as described above. After this crystallization the hexane solution contained mainly $1,3,5$ trimethoxybenzene-2-t. The solvent was stripped off and the solid residue sublimed in vacuo 5-6 times. The last sublimate contained less than 0.7 mole \% product and was used without further purification for the tritium determinations.

Determination of tritium. The determination of the tritium content was carried out as described previously, ${ }^{6}$ but since the isolated substances from the reaction solution contained bromine and probably traces of nitrogen, the combustions to water were performed differently. All combustions, including those of the starting material, were carried out in the following way. The substances were burned in a stream of carbon dioxide with Vinosit $B$ as catalyst, ${ }^{12}$ bromine being removed by silver wool ${ }^{13}$ and nitrogen by freshly reduced copper. ${ }^{12}$

The condition of the measuring unit (Dynacon Model 6000 vibrating reed electrometer connected to a Varian Model G10 recorder) ${ }^{6}$ was checked from time to time by measuring the activity of a large sample of standard tritium water. Nine measurements of the standard water carried out during six months gave a relative standard deviation of $\pm 0.68 \%$.

Six measurements on the sample of $1,3,5$-trimethoxybenzene-2-t gave a relative standard deviation of $\pm 0.80 \%$. This error differs little from the error in the measurements of the standard water and it seems therefore that the error due to possible isotope separation in the combustion step is small. ${ }^{14}$ From the standard deviation obtained for the measurements of the starting material, the error of a single determination is calculated to be $\pm 2 \%$ with $95 \%$ confidence. ${ }^{15}$ This error is assumed to apply also to the determinations of tritium in the recovered starting material and the product. 
The activities were corrected for background, natural decay of tritiurn, the amounts of impurities in the burned substances, the number of hydrogens in the starting material and the product and, when necessary, normalized to the activity of the standard water.

Control experiments on the absence of hydrogen exchange during the reaction and the work-up process. A control "competitive" experiment was carried out with inactive 1,3,5-trimethoxybenzene in dimethylformamide, to which $0.009 \mathrm{ml}$ of tritium water had been added. If equilibration of all exchangeable hydrogens (including those of the water originally present in the solvent, but not those of the solvent itself) had taken place, the remaining 1,3,5-trimethoxybenzene would have had an activity of about 270000 units. The value actually found was less than 18 units. Hydrogen exchange is obviously negligible under these conditions.

A separate check was performed on the repeated sublimations of the recovered starting material. 1,3,5-Trimethoxybenzene-2-t, with some 1-bromo-2,4,6-trimethoxybenzene-3-t added, was sublimed seven times. The recovered 1,3,5-trimethoxybenzene-2-t had an activity of 406 units, while the activity of the original 1,3,5-trimethoxybenzene-2-t was 409 units. This difference is certainly well within the limits of error.

\section{RESULT}

Kinetic observations. In Table 1 some pertinent observations of the reaction rate have been brought together. In these experiments $\mathrm{N}$-bromosuccinimide was rapidly dissolved in thermostated solutions of the substrates.

Considering that the substrates in Table 1 have very different reactivities (see Preparative experiment and de la Mare ${ }^{16}$ ), the substrate seems not to be involved in the rate-determining step.

By also considering the promotive influence of a small amount of added bromine (which immediately reacts with the substrate forming hydrobromic acid) and the inhibitive influence of added silver sulfate (bromide ions being removed), the following possible reaction path may be written:

$\mathrm{N}$-bromosuccinimide $+\mathrm{HBr} \rightarrow$ succinimide $+\mathrm{Br}_{2}$ (rate-determining)

$\mathrm{Br}_{2}+$ substrate $\rightarrow$ product $+\mathrm{HBr}$ (product-determining)

The molecules are to be considered solvated and if possible ionized to a degree depending on the solvating power of dimethylformamide. ${ }^{17}$

Table 1. Observations on the reaction rates with various substrates and added substances.

\begin{tabular}{|c|c|c|c|c|c|}
\hline Substrate & $\begin{array}{r}\text { Conc. of } \\
\text { substrate } \\
\text { M }\end{array}$ & $\begin{array}{l}f \text { Conc. of } \\
\theta \text { N-bromo- } \\
\text { succinimide I }\end{array}$ & $\begin{array}{l}\text { Substance } \\
\text { added }\end{array}$ & $\underset{{ }^{\circ} \mathrm{C}}{\text { Temp. }}$ & $\begin{array}{c}\text { Time for } 50 \% \text { conver- } \\
\text { sion of active bromine } \\
\text { min }\end{array}$ \\
\hline \multicolumn{6}{|l|}{ 1,3,5-Trimethoxy- } \\
\hline benzene & 0.054 & 0.064 & - & $24 \pm 1$ & 4.5 \\
\hline 1) & 0.71 & 0.058 & - & $\overline{1}$ & 3.3 \\
\hline " & 0.082 & 0.046 & chloranil & $"$ & 1.3 \\
\hline$\gg$ & 0.14 & 0.07 & - & $21 \pm 1$ & 6 \\
\hline$"$ & 0.14 & 0.07 & $\begin{array}{l}\text { silver } \\
\text { sulfate }\end{array}$ & " & $>200$ \\
\hline " & 0.14 & 0.07 & bromine & $》$ & $100 \%$ in less than $2 \mathrm{~min}$ \\
\hline $\begin{array}{l}\text { 1-Bromo-2,4,6- } \\
\text { trimethoxybenzene }\end{array}$ & 0.14 & 0.07 & - & ") & 20 \\
\hline Phenol & 0.14 & 0.07 & - & 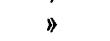 & 4 \\
\hline N-Dimethylaniline & 0.14 & 0.07 & - & 》 & 13 \\
\hline
\end{tabular}

Acta Chem. Scand. 18 (1964) No. 7 
A quite similar mechanism has been experimentally verified for halogenations of reactive substrates with $\mathrm{N}$-haloacetanilides. ${ }^{16}$

All observations, including the absence of inhibitory effect of added chloranil (which is an effective inhibitor for radical reactions ${ }^{18}$ ), are in accord with the suggested polar mechanism (compare also Braude and Waight ${ }^{10}$ ).

The suggested mechanism implies that the total reaction order should be one with respect to $\mathrm{N}$-bromosuccinimide if the concentration of hydrobromic acid is constant during the reaction. The deviation from order one obtained in this case is easily explained by considering that free bromine (which is rapidly converted to hydrobromic acid) is continuously formed in the reaction solution due to decomposition of N-bromosuccinimide. It is possible that this decomposition reaction proceeds by a radical mechanism. It has been observed that free bromine is formed by $\mathrm{N}$-bromosuccinimide in solvents which are not strictly anhydrous, and that the choice of solvent is important. ${ }^{7}$

With 1-bromo-2,4,6-trimethoxybenzene as a substrate it was observed that the reaction solution was somewhat coloured due to free bromine at least at the end of the reaction. It is therefore possible that the first step in the suggested mechanism is not quite rate-determining in this case, which also explains the fact that 1-bromo-2,4,6-trimethoxybenzene reacted slowest among the substrates.

Competitive experiments. The isotope effect $k_{\mathrm{T}} / k_{\mathrm{H}}$, here denoted $\beta$, may be calculated from the ratio $g$ between the molar activity of the remaining and the initial 1,3,5-trimethoxybenzene-2-t, together with the value of the extent of the reaction, denoted $x$. From eqn. $(3-4)$ in "Isotope Effects on Reaction Rates" by Melander ${ }^{19}$ the following function may be deduced: 6

$$
g=(1-x)^{(\beta-1) / 3}
$$

The isotope effect $\beta$ may also be calculated from the ratio $r^{\prime}$ of the molar activity of the product to that of the starting material, together with the extent of the reaction, $x$. Eqn. $(3-14)$ in the text-book of Melander ${ }^{19}$ will in this case be

$$
r^{\prime}=[2 /(\beta+2) x]\left[1-(1-x)^{(\beta+2) / 3}\right]
$$

Both these functions are valid when a molecule, such as 1,3,5-trimethoxybenzene, with three equivalent positions is substituted.

In Figs. 1 and $2, g$ and $r^{\prime}$ have been plotted as functions of $x$ for various values of $\beta$. In the same figures the experimentally found values of $g$ and $r^{\prime}$ have been plotted with their errors. The errors are $\pm 4 \%$ for a single determination of $g$ or $r^{\prime}$ with $95 \%$ confidence (see Determination of tritium) and $\pm 1 \%$ absolute for $x$ (see Analysis). For duplicate values of $g$ or $r^{\prime}$ the total errors are reduced by a factor of $1 / \sqrt{2}{ }^{15}$

From Fig. 1, one obtains $0.97<\beta<1.02$ and from Fig. 2 one obtains $0.87<\beta<1.07$. The limits (95\% confidence) are indicated by dotted curves in the figures.

From inspection of Figs. 1 and 2 it is clear that $g$ is much more sensitive to variation in $\beta$ than $r^{\prime}$ is, and it may be argued that it is not necessary to measure $r^{\prime}$. However, with reactions whose mechanisms and rates are not known in detail, it is judicious to measure both $g$ and $r^{\prime}$ in order to verify that the values of $\beta$ obtained from these do not differ.

* The isotope effect $\beta$ may be related to a single aromatic position, as is done here, if it is assumed that protium reacts at the same rate in molecules containing tritium as in ordinary ones. This assumption is reasonable in the present case. ${ }^{6}$

Acta Chem. Scand. 18 (1964) No. 7 


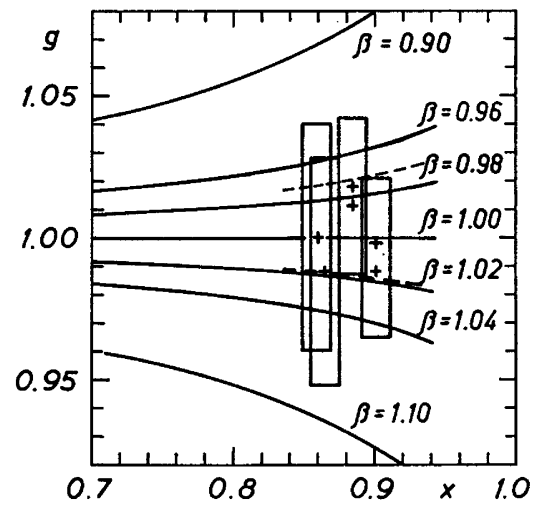

Fig. 1. Relative change in molar activity of 1,3,5-trimethoxybenzene-2-t in the reaction. The curves show $g$ as a function of $x$ for some different values of $\beta$.

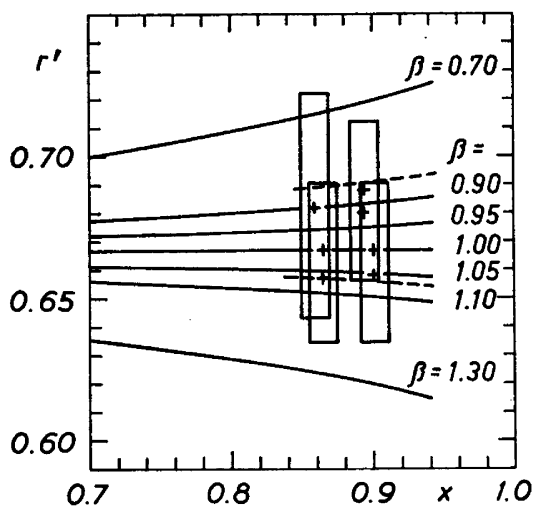

Fig. 2. Molar activity of 1-bromo-2,4,6-trimethoxybenzene-3- $t$ relative to that of initial 1,3,5-trimethoxybenzene-2-t. The curves show $r^{\prime}$ as a function of $x$ for some different values of $\beta$.

Since the values of $\beta$ obtained from $g$ and $r^{\prime}$ do not differ in this case, the more accurate value of $\beta$ may be used and the isotope effect $k_{\mathrm{T}} / k_{\mathrm{H}}$ is thus $1.00 \pm 0.03$ with $95 \%$ confidence.

\section{DISCUSSION}

The observed value of $k_{\mathrm{T}} / k_{\mathrm{H}}$ shows that there is no primary isotope effect in the reaction studied. If the "usual" two-step model of aromatic substitution is employed, it may be concluded that the second reaction step (splitting off of the aromatic hydrogen) is not rate-determining in this case.

Primary isotope effects have been found in azo coupling ${ }^{1}$ and in bromination $^{2-5}$ of sterically hindered aromatic positions. These primary isotope effects have been interpreted in terms of an increase of the potential energy of the transition state in the second reaction step, due to steric hindrance which makes this step rate-determining.

It may be concluded that the steric hindrance in the 1,3,5-trimethoxybenzene molecule is too small to make the second reaction step rate-determining in bromination or azo coupling. ${ }^{6}$

As is usually the case in aromatic substitution, the investigated reaction gave no secondary isotope effect. In this respect the bromination of 1,3,5trimethoxybenzene differs from the azo coupling of the same substrate with $p$-chlorobenzenediazonium ion, which showed a rather pronounced secondary isotope effect, $k_{\mathrm{T}} / k_{\mathrm{H}}$ being larger than one. ${ }^{6}$

Streitwieser ${ }^{20}$ has proposed that the absence of secondary isotope effects in aromatic substitution is due to two effects of rehybridization, which are opposite in direction and about equal in magnitude, viz. a tendency for the 
frequency of the out-of-plane bending mode to increase and a tendency for the same frequency to decrease due to increasing hyperconjugation. Inductive effects are probably of minor importance.

To explain the secondary isotope effect found in the azo coupling reaction one must accordingly assume that hyperconjugation is for some reason of less importance in this case (compare also Olah et al. ${ }^{21}$ ).

In the azo coupling reaction a highly reactive substrate (1,3,5-trimethoxybenzene) reacts with the rather unreactive $p$-chlorobenzenediazonium ion, while in the bromination reaction the same substrate reacts with the much more reactive bromine. Using Hammond's postulate, ${ }^{22}$ one can assume that the rate-determining transition state resembles the initial state more in the bromination reaction than it does in the azo coupling reaction. Accordingly, one would expect less rehybridization in bromination than in azo coupling, which would in turn lead to a smaller secondary isotope effect for the former reaction.

There is certainly more to be done in this field before any decisive conclusions may be drawn. For instance, interactions between a specific reactant and the aromatic hydrogen should probably be taken into account, as well as solvent effects.

The author wishes to thank Professor Lars Melander for his kind advice. This investigation was supported in part by the Swedish Natural Science Research Council. Dr. Robert Carter has kindly revised the English.

\section{REFERENCES}

1. Zollinger, H. Helv. Chim. Acta 38 (1955) 1597, 1617, 1623.

2. Farell, P. G. and Mason, S. F. Nature 183 (1959) 250.

3. Myhre, P. C. Acta Chem. Scand. 14 (1960) 219.

4. Baciocchio, E., Illuminati, G. and Sleiter, G. Tetrahedron Letters 23 (1960) 30.

5. Christen, M. and Zollinger, H. Helv. Chim. Acta 45 (1962) 2066.

6. Helgstrand, E. and Lamm, B. Arkiv Kemi 20 (1962) 193.

7. Horner, L. and Winkelmann, E. H. Angew. Chem. 71 (1959) 349.

8. Ross, S. D., Finkelstein, M. and Petersen R. C. J. Am. Chem. Soc. 80 (1958) 4327.

9. Schmid, H. Helv. Chim. Acta 29 (1946) 1144.

10. Braude, E. A. and Waight, E. S. J. Chem. Soc. 19521116.

11. Hesse, O. Ann. 276 (1893) 328.

12. Halvarson, K. and Melander, L. Arkiv Kemi 11 (1957) 77.

13. Halvarson, K. and Melander, L. Arkiv Kemi 8 (1956) 29.

14. Melander, L. Arkiv Kemi 2 (1950) 211.

15. Laitinen, H. A. Chemical Analysis, McCraw-Hill Book Co., New York 1960, p. 546.

16. De la Mare, P. B. D. and Ridd, J. H. Aromatic Substitution, Butterworths Scientific Publications, London 1959, pp. 140, 151.

17. Smith, S. G., Fainberg, A. H. and Winstein, S. J. Am. Chem. Soc. 83 (1961) 618.

18. Ford, M. C. and Waters, W. A. J. Chem. Soc. 19522240.

19. Melander, L. Isotope Effects on Reaction Rates, Ronald Press Co., New York 1960, pp. 51, 56 .

20. Streitwieser, A., Jr.. Jagow, R. H., Fahey, R. C. and Suzuki, S. J. Am. Chem. Soc. 80 (1958) 2326.

21. Olah, G. A., Kuhn, S. J. and Flood, S. H. J. Am. Chem. Soc. 83 (1961) 4571, 4581.

22. Hammond, G. S. J. Am. Chem. Soc. 77 (1955) 334.

Received May 28, 1964. 